\title{
Priorités de la recherche forestière au Canada pour 1993
}

\author{
Survol à l'intention du Conseil canadien des ministres des forêts \\ rédigé par le Conseil consultatif de la recherche forestière du Canada en collaboration avec les comités \\ consultatifs de la recherche forestière des provinces et des territoires
}

Ottawa, juillet 1993

\section{Résumé}

Avec la collaboration des comités consultatifs de la recherche forestière des provinces et des territoires (CCRFPT), le Conseil consultatif de la recherche forestière du Canada (CCRFC) a rédigé son cinquième exposé annuel des priorités de la recherche forestière, destiné au Conseil canadien des ministres des Forêts (CCMF).

Les cinq priorités de chaque comité ont reçu, dans l'ordre décroissant, la note de 5 à 1 . Nous avons ainsi cerné 11 sujets de recherche dont l'ordre décroissant de priorité est le suivant :

1. systèmes intégrés d'aménagement des paysages forestiers et aide à la décision ;

2. inventaire, accroissement et rendement, classification écologique des stations ;

3. fonctionnement des écosystèmes, conservation des sols et de la diversité biologique ;

4. gestion raisonnée des ravageurs et des mauvaises herbes ainsi que solutions de rechange aux produits chimiques ;

5. effets écologiques des perturbations naturelles et anthropiques ;

6. sylviculture et récolte à moindre coût ; régénération naturelle ;

7. prévention et extinction des incendies de forêt ;

8. connaissances socio-économiques et résolution des conflits d'utilisation des ressources ;

9. aménagement des peuplements mixtes ;

10. encouragement aux investissements privés ;

11. qualité du matériel de plantation et amélioration des arbres.

Huit questions susceptibles d'influer sur les priorités à venir de la recherche ont été mentionnées par au moins deux comités, comme suit :

1. les échanges commerciaux, la concurrence et l'opinion publique à la grandeur de la planète ;

2. les préoccupations et les nouvelles données concernant la foresterie durable et l'environnement ;

3. la participation du public ;

4. les nouvelles politiques concernant la foresterie, les tenures, les ventes et la protection des forêts ;

5. l'absence, à long terme, de financement, de communications et de coordination pour la recherche ;

6. la pénurie de fibres, la viabilité à long terme de l'industrie ;

7. les revendications territoriales et la foresterie sur les terres des autochtones ;

8. le besoin croissant d'information à tous les niveaux.

Parmi les autres sujets de préoccupation, citons le changement climatique, l'endettement de l'État, la croissance démographique et les déplacements de populations, les forêts protégées et les vieilles forêts.
La discussion de ces priorités et enjeux au cours d'une réunion du CCRFC et des représentants des comités provinciaux et territoriaux a permis d'arriver à certaines conclusions générales, que nous exposons ci-dessous.

Pour résoudre les conflits d'utilisation des ressources et pour aménager la durabilité des forêts afin que tous en tirent avantage, les aménagistes ont besoin de meilleurs renseignements, que seule la recherche peut leur fournir. On vise à obtenir des systèmes d'aménagement qui procureront toute une gamme de produits de la forêt et qui maintiendront la diversité et l'hétérogénéité des écosystèmes forestiers naturels. Ce type de foresterie satisfera aux désirs de la plupart des Canadiens et contribuera énormément à restaurer la confiance du public à l'égard de l'aménagement forestier.

Bref, la recherche doit fournir des connaissances supplémentaires aux aménagistes pour que ces derniers puissent :

1. maintenir la diversité et la résilience des écosystèmes forestiers ;

2. améliorer la santé des forêts et leur résistance aux ravageurs ;

3. augmenter la productivité forestière à tous les points de vue ;

4. réduire les coûts de l'aménagement forestier et de la protection contre le feu ;

5. accéder à toutes les connaissances et données disponibles pour la prise des décisions ;

6. déterminer la valeur socio-économique des forêts canadiennes ;

7. augmenter les profits et la compétitivité dans l'industrie forestière.

Beaucoup estiment que la recherche forestière au Canada est au diapason de ces questions, mais il faut tendre davantage à assurer un financement à long terme suffisant et à améliorer l'application locale de l'acquis de la recherche.

Le présent relevé annuel des priorités de la recherche s'est révélé utile. On a en effet conclu à la grande utilité de la rencontre annuelle entre le CCRFC et les représentants des comités consultatifs de la recherche des provinces et des territoires ainsi que de la présentation annuelle du rapport au CCMF. Toutefois, l'expérience montre qu'il n'est pas nécessaire de faire un relevé annuel des priorités. On recommande un examen bisannuel, avec présentation au CCMF et publication ultérieure. Pour les années intercalaires, on propose un atelier du CCRFC et des représentants des comités provinciaux et territoriaux pour examiner en profondeur un sujet important de la recherche et présenter ses résultats et ses conclusions au CCMF. S'il y a lieu, on publiera un rapport.

\section{Introduction}

Le CCRFC a préparé pour le CCMF ce cinquième relevé annuel des priorités de la recherche avec la collaboration 
des comités consultatifs de la recherche forestière des provinces et des territoires. Les priorités définies par ces comités constituent le point de départ de ces relevés, qui sont présentés chaque année aux ministres, à leur réunion automnale, puis publiés dans The Forestry Chronicle. Le présent relevé se fonde sur des renseignements fournis au début de 1993.

Chaque comité a répondu aux questions suivantes :

1. énumérez et décrivez très succinctement vos cinq priorités de recherche forestière dans l'ordre décroissant de l'urgence que vous attachez à chacune en réponse à la question suivante : " Dans votre région, quels sont les occasions ou les problèmes de foresterie les plus importants pour lesquels il faut élargir l'effort de recherche et l'appui à la recherche afin d'améliorer l'aménagement forestier de façon que toute la palette des avantages pour la forêt puisse en découler de façon durable ? » La liste de sujets proposée ci-dessous n'est donnée qu'à titre indicatif. N'hésitez pas à ajouter d'autres sujets, mais n'en mentionnez que cinq. À noter que, en vertu d'une décision prise l'année dernière, ce survol des priorités n'inclut pas celles de la recherche sur les produits forestiers.

2. Décrivez toute nouvelle question sociale, environnementale et économique susceptible d'influer sur les pratiques forestières et les priorités de la recherche.

3. Donnez votre opinion sur la qualité et l'adéquation de la recherche forestière dans votre région. (Cette question ne porte pas sur l'ampleur de la recherche ni sur le caractère suffisant du financement et de l'appui.)

Les réponses ont été envoyées au secrétaire de direction du CCRFC, qui en a dressé les tableaux de synthèse, en vue d'un examen et de discussions à une réunion du Conseil consultatif et des représentants des comités des provinces et des territoires, les 12 et 13 mai 1993, à Ottawa. D'après ces discussions, un rapport a été rédigé, diffusé pour observations, puis révisé pour être présenté au CCMF.

\section{Priorités de la recherche des comités des provinces et des territoires}

À partir des réponses fournies sur les priorités, on a dressé un tableau des sujets, que l'on a amalgamés s'il y avait lieu, pour obtenir la liste des 11 sujets de recherche identifiés par au moins un comité consultatif dans sa liste des 5 priorités. Le tableau 1 résume ce travail de synthèse. Les grandes priorités ont reçu la note de 5 ; les suivantes, 4 et ainsi de suite. Les sujets sont présentés dans l'ordre décroissant de leur priorité, établi selon ces pointages. Dans certaines provinces ou territoires, le nombre de sujets est inférieur à cinq parce que deux des sujets ont été amalgamés ; les pointages ont été corrigés en conséquence.

Les sujets ne sont pas neufs, mais le libellé, le groupement des notions et l'accent qui en résulte diffèrent de ceux des années antérieures. Il semble que les priorités de la recherche ne changent pas trop rapidement pour la capacité d'adaptation des organismes de recherche.

Ces priorités sont analysées dans les paragraphes qui suivent, dans l'ordre décroissant de l'importance qu'on leur attache.
1. Systèmes intégrés d'aménagement des paysages forestiers et aide à la décision

Pour la deuxième année, ce sujet se classe au premier rang. Les pressions du public pour maintenir les zones sauvages et les zones de loisirs rendent l'aménagement intégré des forêts nécessaire. Les aménagistes forestiers ont trop souvent voulu maximiser la possibilité annuelle plutôt que définir les types de paysages forestiers et leur répartition nécessaires à la réalisation des avantages recherchés.

On a besoin de systèmes améliorés d'aide à la décision afin d'utiliser de façon efficace la masse complexe de données que l'on tire de l'examen de tous les facteurs. Il faut des systèmes experts à la portée de l'utilisateur pour permettre aux aménagistes de réunir tous les renseignements utiles aux meilleurs choix en matière d'aménagement forestier et d'utilisation des sols ainsi que d'évaluer les compromis nécessaires.

\section{Inventaire, accroissement et rendement,} classification écologique des stations

Pour procurer tous les avantages, l'aménagement des paysages forestiers devrait reposer sur une base plus solide. Il exige des méthodes d'inventaire améliorées qui ne permettent pas seulement d'évaluer le bois, mais aussi la faune, les débits des cours d'eau, les loisirs et d'autres valeurs. En outre, on a besoin de classifications coordonnées des écosystèmes, de sorte que les systèmes d'aménagement acquis de la recherche effectuée dans un écosystème particulier puissent, après évaluation convenable, être adaptés en vue de leur application ailleurs.

\section{Fonctionnement des écosystèmes, conservation des} sols et de la diversité biologique

Il est indispensable de connaître la " mécanique » des écosystèmes forestiers si l'on veut en faire l'aménagement durable. Cette exigence ne peut pas être séparée d'autres priorités qui montrent la nécessité de systèmes intégrés d'aménagement des paysages forestiers, d'inventaires précis de toutes les valeurs, d'écosystèmes forestiers en santé et d'une évaluation soignée des effets des perturbations sur ces derniers. En outre, il faut de la recherche pour aider à préciser le degré de diversité biologique requis pour maintenir l'écosystème forestier.

\section{Gestion raisonnée des ravageurs et des mauvaises herbes} ainsi que solutions de rechange aux produits chimiques

On a besoin de meilleurs moyens de maintenir et d'améliorer la santé générale des forêts : méthodes améliorées de lutte contre des insectes, des maladies et de la végétation concurrente bien précis, qui prélèvent un lourd tribut d'arbres et retardent l'accroissement. Pour aider à évaluer la nécessité de mesures de lutte, on a besoin de meilleurs moyens d'évaluer le risque de dégâts graves attaché aux proliférations décelées dans leurs premiers stades. Des solutions de rechange aux produits chimiques sont nécessaires en réaction à la demande du public, mais leurs risques et avantages doivent être soigneusement évalués, et de nouvelles méthodes devraient être trouvées pour que ce but soit atteint de façon plus efficace. Tout aussi importants toutefois sont le maintien d'une forêt en meilleur état et la réduction du besoin de mesures précises de lutte, qui découlent de la recherche visant à aménager le paysage forestier d'une façon intégrée et durable, comme cela est exposé dans les autres priorités. 
Tableau 1. Priorités de la recherche, 1993

Sujets prioritaires de la recherche forestière T.-N. N.-É. Î.P.-É. N.-B. Qc Ont. Man. Sask. Alb. C.-B. Yukon T.N.-0. Pointage

1. Systèmes intégrés d'aménagement des paysages forestiers et aide à la décision

2. Inventaire, accroissement et rendement, classification écologique des stations

3. Fonctionnement des écosystèmes, conservation des sols et de la diversité biologique

4. Gestion raisonnée des ravageurs et des mauvaises herbes ainsi que solutions de rechange aux produits chimiques

5. Effets écologiques des perturbations naturelles et anthropiques

6. Sylviculture et récolte à moindre coût ; régénération naturelle

7. Gestion, prévention et répression des incendies de forêt

8. Connaissances socio-économiques et résolution des conflits d'utilisation des ressources

9. Aménagement des peuplements mixtes

10. Encouragement aux investissements privés

11. Qualité du matériel de plantation et amélioration des arbres

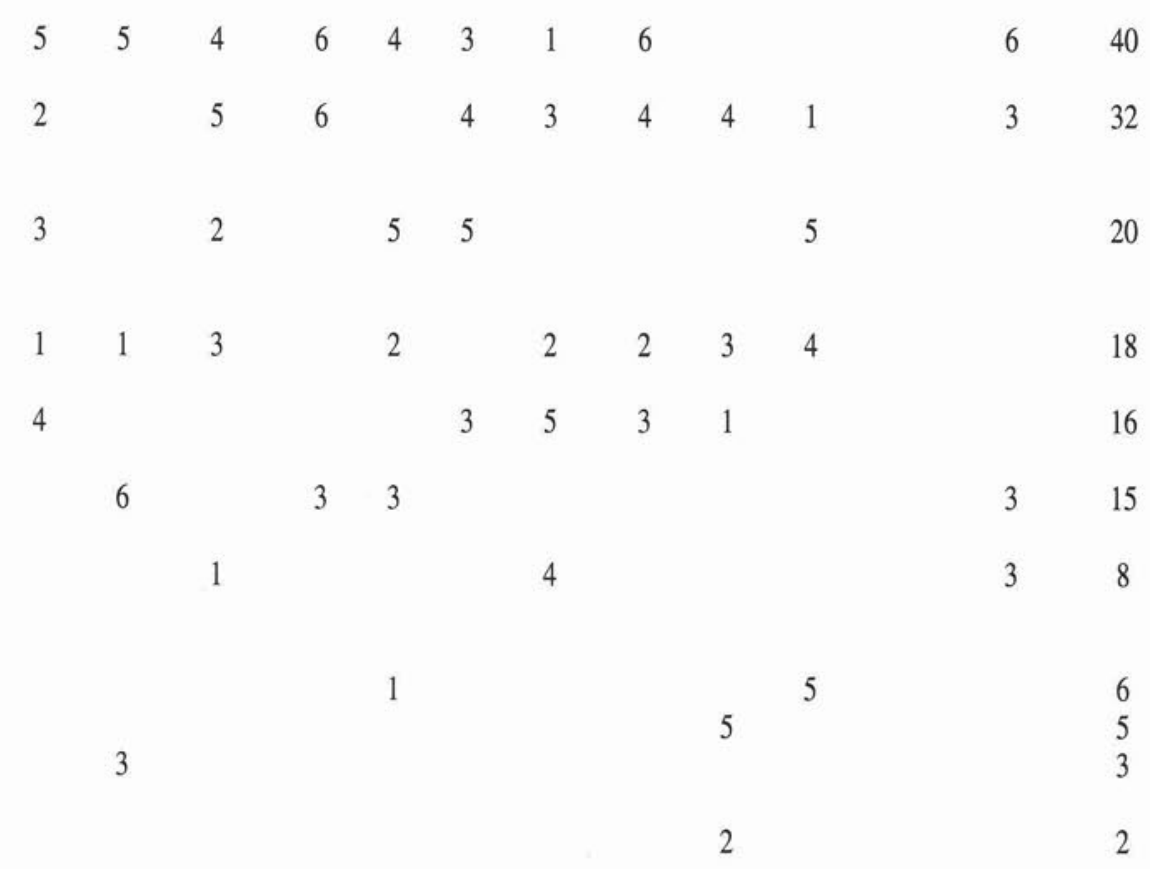

Nota - Le Nouveau-Brunswick et les Territoires du Nord-Ouest accordent une priorité égale aux cinq sujets. Un pointage de 3 a été attribué aux sujets choisis. Quand le pointage dépasse 5, c'est que certains sujets ont été amalgamés.

5. Effets écologiques des perturbations naturelles et anthropiques

Les effets à long terme, sur les écosystèmes forestiers, des pratiques forestières et de la récolte, du changement climatique et des perturbations naturelles telles que le feu et les pullulations de ravageurs ont besoin d'être mieux compris afin d'en assurer la durabilité. Les donnés zéro proviendront de l'atteinte de la priorité numéro 2 , mais cette information doit être suivie d'une surveillance à long terme pour déceler et enregistrer les changements.

\section{Sylviculture et récolte à moindre coût ; régénération naturelle}

La réduction des coûts de la sylviculture et de la récolte est une priorité, en partie à cause du climat économique morose. Toutefois, le besoin, depuis longtemps reconnu, de corréler la récolte à la régénération et l'attrait, pour les écologistes, des systèmes de sylviculture et de régénération plus naturels et moins perturbateurs sont irrésistibles. La régénération naturelle (par opposition à la plantation), le désherbage à la main (par opposition aux herbicides) et la polyculture (par opposition à la monoculture) sont autant d'exemple de ces systèmes.

\section{Prévention et extinction des incendies de forêt}

Les incendies de forêt provoquent des dégâts très graves. Chaque année, la superficie brûlée dépasse très légèrement le million d'hectares, et, dans les années où les forêts sont gravement touchées, les pertes s'alourdissent rapidement. Le réchauffement planétaire peut aggraver la situation des incendies de forêt. Le phénomène $a$ donc besoin d'être rigoureusement étudié. Grâce à l'ordinateur, qui intègre l'information et qui gère les ressources affectées à l'extinction, on a considérablement amélioré la maîtrise du feu, mais la poursuite de la recherche pour améliorer ces méthodes et élargir leur application sera très rentable.

8. Connaissances socio-économiques et résolution des conflits d'utilisation des ressources

Les forêts du Canada sont de plus en plus en demande, pour tous les types d'utilisation. Les disputes pour les ressources forestières telles que l'eau pure, la faune, les paysages pittoresques et la nature sauvage ont abouti à des demandes de report ou d'exclusion de la coupe de bois. Trop souvent hélas, ces demandes sont formulées sans une compréhension suffisante des répercussions sociales et économiques. Pour aider à résoudre ces différends, on a besoin d'effectuer de la recherche dans le domaine des sciences sociales, économiques et des sciences du comportement dans leur rapport avec les questions de ressources forestières.

\section{Aménagement des peuplements mixtes}

C'est là la priorité numéro un de l'Alberta en 1993. En effet, cette province possède de vastes forêts mixtes, et, dernièrement, l'industrie forestière s'est développée en fonction de cette richesse. L'aménagement des écosystèmes de forêts mixtes préoccupe également l'est du Canada.

\section{Encouragement aux investissements privés}

Avec la diminution du financement de l'état, les soins sylvicoles et l'aménagement sont menacés de diminuer à moins que l'on ne trouve des moyens de stimuler l'investissement. Cela est particulièrement important en Nouvelle-Écosse, où la plus grande partie du territoire forestier appartient à des intérêts privés. 


\section{Qualité du matériel de plantation et amélioration} des arbres

Malgré la tendance très forte vers des pratiques forestières plus discrètes et malgré l'affectation d'un territoire plus étendu aux loisirs, à la nature sauvage et à d'autres utilisations notées sous la priorité 8 , il importe de pouvoir augmenter les rendements en bois sur certains terrains exploitables de façon intensive. Une régénération plus rapide et plus efficace à l'aide d'un matériel amélioré ainsi que des soins sylvicoles et des mesures de protection améliorés sont des moyens d'atteindre ce but.

\section{Questions susceptibles de modifier les priorités de la recherche}

Huit questions ont été désignées, par au moins deux comités, comme susceptibles de modifier les priorités à venir de la recherche.

1. Les échanges commerciaux, la concurrence et l'opinion publique à la grandeur de la planète.

2. Les préoccupations et nouvelles données concernant la foresterie durable et l'environnement.

3. La participation du public.

4. Les nouvelles politiques concernant la foresterie, les tenures, les ventes et la protection des forêts.

5. L'absence, à long terme, de financement, de communications et de coordination pour la recherche.

6. La pénurie de fibres, la viabilité à long terme de l'industrie.

7. Les revendications territoriales et la foresterie sur les terres des autochtones.

8. Le besoin croissant d'information à tous les niveaux.

Les préoccupations formulées à l'égard des échanges commerciaux à l'échelle du globe, de la foresterie durable et de l'environnement de même que de la contribution du public à ces questions ont été évoquées par beaucoup de comités. Ces préoccupations se traduisent par la priorité accordée à la mise au point de systèmes intégrés d'aménagement des paysages forestiers, à la compréhension des effets écologiques des diverses causes de perturbation des forêts et à la recherche de moyens d'améliorer la santé des forêts et de trouver des solutions de rechange aux pesticides chimiques. De même, la demande d'une recherche plus intense sur le fonctionnement des écosystèmes forestiers et sur la mise au point, s'il y a lieu, de techniques différentes d'aménagement forestier, moins perturbatrices, découle de ces préoccupations.

Parmi les autres questions mentionnées par un seul comité, citons le changement climatique, l'endettement de l'État, l'accroissement et les mouvements démographiques, les forêts protégées et les vieilles forêts ainsi que l'absence de vision stratégique pour nos forêts et le secteur forestier.

\section{Survol et synthèse}

Ces priorités et ces enjeux ont fait l'objet d'une discussion approfondie à la réunion de mai du CCRFC et des représentants des provinces et des territoires. Il s'en est dégagé certaines conclusions générales, décrites ci-dessous.

Le défi auquel font face les aménagistes forestiers est de résoudre les différends centrés sur l'utilisation des ressources et l'aménagement de la durabilité des forêts du Canada pour en tirer tous les avantages et, en même temps, préserver leur diversité biologique. À cette fin, ils ont besoin de savoir comment planifier et mettre en œuvre des systèmes d'aménagement forestier qui intègrent toutes les valeurs et qui reconnaissent la structure naturellement hétérogène des écosystèmes forestiers. Cette vision stratégique de la foresterie satisfera aux désirs d'un grand nombre de Canadiens et contribuera énormément à restaurer la confiance du public à l'égard de l'aménagement forestier.

Plus précisément, on a besoin de connaissances pour :

1. maintenir la diversité et la résilience des écosystèmes forestiers ;

2. améliorer la santé des forêts et leur résistance aux ravageurs ;

3. augmenter la productivité forestière à tous les points de vue ;

4. pratiquer un aménagement forestier plus efficace relativement aux coûts, y compris la gestion des incendies ;

5. accéder à toute la gamme des connaissances et des données disponibles pour les décisions à prendre concernant l'utilisation des terres et l'aménagement des ressources ;

6. évaluer les avantages socio-économiques de la ressource forestière et la valeur que leur accordent les Canadiens ;

7. assurer la profitabilité et la compétitivité de l'industrie forestière.

Au cours de ces survols on s'est beaucoup inquiété de la négligence des priorités de la recherche sur les produits forestiers. La demande mondiale de produits forestiers est susceptible d'augmenter, de nouveaux débouchés peuvent être trouvés, mais il y aura également la concurrence des nouveaux producteurs. La transformation améliorée du bois, la mise au point de produits à valeur ajoutée pour utiliser au mieux les stocks actuels et à venir, la réduction de la demande d'énergie et le recyclage sont tous des moyens qui seront très importants pour que le bois reste un matériau concurrentiel et pour que le Canada soit davantage capable de tirer parti de son riche capital de ressources forestières. On en a conclu que, à l'avenir, les survols devraient s'attacher davantage aux priorités de la recherche sur les produits forestiers.

\section{Adéquation et qualité de la recherche forestière}

Les opinions sur l'adéquation et la qualité de la recherche ont beaucoup plus varié en 1993 qu'au cours des années antérieures. Plusieurs comités (ColombieBritannique, Nouvelle-Écosse et Terre-Neuve) n'ont pas répondu à cette question, mais l'opinion des autres a varié du très critique au positif.

À l'Alberta, la recherche forestière semble polarisée, mal coordonnée avec l'industrie, dépourvue de stratégie à long terme, orientée vers le court terme, dénuée de valeurs scientifiques et opérationnelles bien définies et sans méthode uniforme acceptée d'évaluation des projets.

Pour la Saskatchewan, la qualité et l'adéquation de la recherche augmentent, tandis que les rapports plus étroits avec l'État, l'industrie ainsi qu'avec les groupes d'écologistes et les groupes spéciaux de pression aident à attirer l'attention des chercheurs sur des problèmes précis. 
Pour le Manitoba, la qualité de la recherche forestière est considérée comme excellente, et beaucoup de projets de recherche entrepris en vertu de l'entente fédérale-provinciale produisent déjà des résultats préliminaires. Toutefois, certaines zones critiques restent sous-financées ou tout à fait négligées.

Les observations de l'Ontario ont porté exclusivement sur le programme du ministère des Richesses naturelles de l'Ontario. La qualité et l'adéquation de la recherche s'améliorent, et le financement de l'initiative Forêts durables a permis à la province d'embaucher des scientifiques supplémentaires. Les centres régionaux de sciences et de technologie visent à livrer des renseignements sur la recherche aux clients sur le terrain. Leur action a été renforcée grâce à un financement accru et à l'élargissement de la gamme des spécialistes.

Le Conseil consultatif québécois signale que la plupart des programmes de recherche-développement sont pertinents, puisqu'ils insistent sur des questions très prioritaires, qui font problème ; il signale cependant qu'il faut accroître le financement de la recherche forestière en raison de l'importance socio-économique du secteur.

La situation au Nouveau-Brunswick est décrite comme excellente pour ce qui concerne l'identification des besoins de la recherche et la qualité de cette dernière. On insiste sur les programmes de coopération, lesquels expliquent dans une grande mesure les réalisations réussies.

Le comité de l'île-du-Prince-Édouard s'est dit inquiet de la dépendance actuelle à l'égard du Plan vert et des fonds de l'extérieur pour appuyer la recherche. Cette dépendance augure mal pour l'appui fourni à la recherche à long terme exigée pour le développement durable.

D'après le comité des Territoires du Nord-Ouest, la qualité de la recherche est convenable, des efforts étant faits pour mieux faire comprendre les programmes de recherche et donner aux collectivités l'occasion de participer.

\section{Conclusion}

À la réunion de mai 1993, on a discuté de l'utilité de ce relevé annuel des priorités de la recherche. On a conclu que la réunion annuelle du CCRFC avec les représentants des comités consultatifs de la recherche forestière des provinces et des territoires était très utile, tout comme la présentation annuelle du rapport au CCMF. Toutefois, il n'est pas utile de préparer un relevé annuel de ces priorités, à la lumière de l'expérience des cinq dernières années. En effet, les priorités de la recherche en 1993 ne sont pas très différentes de celles de 1992. On en conclut que le survol des priorités devrait être effectué à tous les deux ans et qu'il serait présenté au CCMF, puis publié comme à l'accoutumé. Au cours des années intercalaires, on devrait organiser un atelier auquel participeraient le CCRFC ainsi que les représentants des comités des provinces et des territoires, pour s'attacher à un sujet important de recherche, et on devrait présenter les résultats et les conclusions de ce travail au CCMF. Si on le juge utile, on pourrait publier un rapport sur l'atelier.

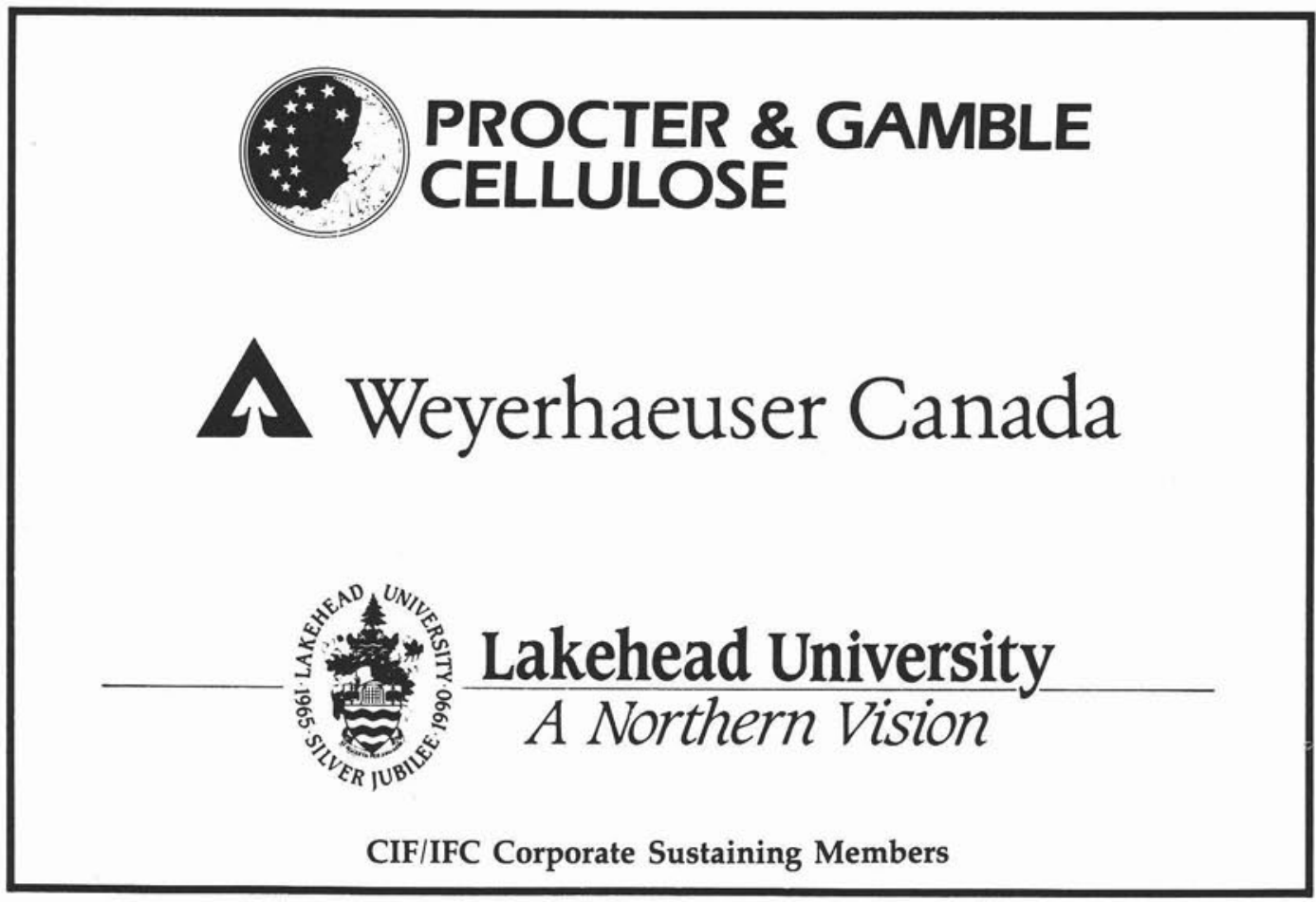

